\title{
Understanding the performance of atmospheric free-space laser communications systems using coherent detection
}

\author{
Aniceto Belmonte \\ Technical University of Catalonia, Department of Signal Theory and Communications \\ 08034 Barcelona, Spain \\ Joseph M. Kahn \\ Stanford University, Department of Electrical Engineering \\ Stanford, CA 94305, USA
}

\begin{abstract}
We introduce a realistic model for the impact of atmospheric phase and amplitude fluctuations on free-space links using either synchronous or nonsynchronous detection. We compare options for atmospheric compensation, including active modal methods and diversity combining techniques. We consider the effects of log-normal amplitude fluctuations and Gaussian phase fluctuations, in addition to local oscillator shot noise. We study the effect of various parameters, including the ratio of receiver aperture diameter to wavefront coherence diameter, the scintillation index, the number of modes compensated, and the number of independent diversity branches combined at the receiver. We analyze outage Shannon capacity, placing upper bounds on the achievable spectral efficiency and enabling the performance of specific system designs to be predicted.
\end{abstract}

Keywords: Atmospheric turbulence; Statistical optics; Adaptive optics; Optical communications.

\section{INTRODUCTION}

Evaluating the performance of a heterodyne receiver in the presence of atmospheric turbulence is generally difficult because of the complex ways turbulence affects the coherence of the received signal that is to be mixed with the local oscillator $[1,2]$. Light propagated through a turbulent atmosphere contains speckle which will be present at the detector surface. Therefore, illuminating a single-element detector with a uniform LO beam will produce mismatch of the amplitudes and phases of the two fields resulting in a loss in downconverted power (see Fig. 1). The downconverted heterodyne power is maximized when the spatial field of the received signal matches that of the local oscillator.

The use of adaptive compensation of atmospheric wave-front phase distortions to improve the performance of atmospheric systems has been an important field of study for many years. Phase-compensated receivers offer the potential for overcoming atmospheric limitations by adaptive tracking of the beam wave-front and consequent correction of atmospherically induced aberrations. In particular, the modal compensation method involves correction of several modes of an expansion of the total phase distortion in a set of basis functions [3]. As an alternative to a single monolithic-aperture coherent receiver with a full-size collecting area, a large effective aperture can be achieved by combining the output signal from an array of smaller receivers (see Fig. 1). The advantage of a coherent array in terms of the coupling efficiency is that the number of turbulence speckles over each subaperture in the array is much smaller than it would be over a single large aperture. Because each receiver can now be smaller than the scale on which the signal wavefront varies, the local oscillator phase can be matched to the signal to achieve effective coherent reception. Output signals from these receivers can then be combined electronically to improve the detection statistics. In general, the performance of a combined system should improve with an increasing number of receivers and, consequently, given a fixed collecting area, the combined system can offer superior performance. It is important to note that diversity combining consists of receiving redundantly the same information signal over two or more fading channels and to exploit the low probability of concurrence of deep fades in all the diversity channels. We define a general model for the output SNR of diversity systems over correlated fading channels. For systems where the atmospheric fading on the branches is correlated or dependent, we can solve the problem by transforming it into an independent problem using the technique of spatial whitening. 


\section{PERFORMANCE OF COHERENT RECEIVERS}

We have studied in a unified framework the effects of both phase-front distortion and amplitude scintillation on the performance of synchronous (coherent) receivers utilizing phase-front compensation and diversity-combining techniques. First, we have defined a mathematical model for the received signal after propagation through the atmosphere [3]. By noting that the downconverted signal current can be characterized as the sum of many contributions from different coherent regions within the aperture, we have shown that the probability density function (PDF) of this current can be well-approximated by a modified Rice distribution (Fig. 3). In our model, the parameters describing the PDF depend on the turbulence conditions and the degree of modal compensation applied to the receiver.

Second, we analyze how diversity-combining of several replicas of the transmitted signal, each corrupted independently by the atmosphere, can provide more reliable communication because of the low probability of deep fades in all the diversity channels [5]. We consider two types of receive diversity combining. First we assume the receiver has knowledge of the instantaneous channel state, making perfect maximal ratio combining (MRC) diversity possible. In this diversity scheme, the receiver co-phases the intermediate signals, adjusts their amplitudes separately, and sums them to obtain a composite signal with improved SNR. The rate at which phase and amplitude must be adjusted will be dictated by the rate at which the atmospheric turbulence fluctuates, generally no higher than $1 \mathrm{kHz}$. The MRC receiver is the optimal combining technique in that it yields a carrier with the highest mean SNR and lowest SNR fading. The optimum electronic gain for each receiver should be proportional to the received signal field amplitude. Note that when the electronic gains and phase delays are back-propagated into the LO, the optimum gain and phase adjustments would result in an amplitude and phase match of this synthetic LO field to the distorted signal field. For independent branch signals and equal average branch SNR, the PDF of the received SNR at the output of a perfect $L$-branch MRC coherent combiner in the atmosphere would be described a sum of $L$ independent and identically distributed non-central chisquared random variables with two degrees of freedom (see Fig. 3). This random variable has a noncentral chi-square distribution with $2 L$ degrees of freedom 6 .

Then we consider the case when selective combining (SC) diversity schemes is used. A selection receiver is quite different from the coherent MRC summing receiver as the single strongest intermediate signal is switched to the output, while all the other array element signals are discarded. Although this simple diversity technique clearly does little to improve the mean SNR value of the collected signal, it does provide a reduction in the signal fading. Assuming independent branch signals and equal average branch SNR, the outage probability of the received signal photons at the output of an $L$-branch SC coherent combiner is found to be given by the $L$ th power of the CDF corresponding to a single-branch receiver $[5,6]$.

In this work, we analyze the spectral efficiency of optical communication over the clear turbulent atmosphere. Defined as the average transmitted data rate per unit bandwidth for a specific average transmit power and probability of outage or error, achievable spectral efficiency is an important performance measure for free-space optical communication systems [7]. As the use of multilevel phase modulation schemes increase spectral efficiency by sending multiple bits per symbol, optical coherent reception provides an alternative to direct detection schemes for free-space optical communication applications. Figures 3-6 show the results of this analysis.

Figures 3 and 4 analyze the use of adaptive compensation of atmospheric wave-front phase distortions. We study the $\varepsilon$ outage capacity for different outage probabilities $\varepsilon$ as a function of the average turbulence free SNR $\gamma_{0}$, the receiver aperture diameter $D$, the number of spatial modes $J$ removed by the compensation system, and the strength of atmospheric turbulence. Turbulence is quantified by two parameters: the phase coherence length $r_{0}$ [1] and the scintillation index $\sigma_{\beta}{ }^{2}[8,9]$. Figure 3 presents the $\varepsilon$-outage spectral efficiency vs. turbulence-free SNR $\gamma_{0}$. Figure 3(a) shows the capacity for different values of the normalized aperture diameter $D / r_{0}$, while Fig. 3(b) shows the performance for different values of $J$, the number of modes compensated. In all cases, we use a small outage probability $\varepsilon=0.001$. We assume no scintillation, $\sigma_{\beta}{ }^{2}=0$, so the effect of turbulence is simply to reduce the coherence length $r_{0}$. For a fixed aperture diameter $D$, as $r_{0}$ is reduced, the normalized aperture diameter $D / r_{0}$ increases, and turbulence reduces the heterodyne or homodyne downconversion efficiency. Just using a normalized aperture diameter $D / r_{0}=10$, turbulence reduces the capacity of the atmospheric channel to very small values for all values of the SNR $\gamma_{0}$ considered. When phase correction is used, as in Fig. 3(b), in most situations compensation of just a few modes yields a substantial performance improvement. Compensation of $J=20$ modes yields significant improvement for even the largest normalized apertures considered. For example, for a normalized aperture $D / r_{0}=10$, the 0.001 -outage spectral efficiency can be as large as $8 \mathrm{bits} / \mathrm{s} / \mathrm{Hz}$ for the higher values of SNR $\gamma_{0}$ considered. 
Figure 4 considers the effect of aperture diameter on the $\varepsilon$-outage spectral efficiency. It presents the spectral efficiency as a function of the normalized aperture $D / r_{0}$ for a constant phase coherence length $r_{0}$. For the smallest aperture diameter considered, the turbulence-free SNR has a value $\gamma_{0}=10 \mathrm{~dB}$. For any other aperture diameter, the value of $\gamma_{0}$ is proportional to $D^{2}$. Figure 4 (a) presents capacity for different values of the outage probability $\varepsilon$. The dependence on scintillation index $\sigma_{\beta}{ }^{2}$ is very weak, as it can be seen in Fig. 4(a) for $\varepsilon=0.001$, where the outage capacity for $\sigma_{\beta}{ }^{2}=0$ (solid line) and $\sigma_{\beta}{ }^{2}=1$ (dashed line) are compared. In Fig. 4(a), the existence of an optimal aperture diameter in coherent freespace links is apparent. This optimal aperture diameter maximizes the $\varepsilon$-outage capacity. When the aperture is larger than the optimal value, phase distortion cannot be overcome by the increase on collected power and we observe a decrease in capacity. When larger normalized apertures $D / r_{0}$ are considered in Fig. 4(a), the capacity tends toward an asymptotic value that is independent of normalized aperture diameter $D / r_{0}$. In Fig. 4(b), we consider a small outage probability $\varepsilon=0.001$ and show the $\varepsilon$-outage spectral efficiency for different values of $J$, the number of modes compensated. As we increase $J$, the optimized value of $D / r_{0}$ increases, and the optimized capacity improves appreciably. Even for such small outage probability, with compensation of $J=20$ modes, and optimized $D / r_{0}$, an outage spectral efficiency of $14 \mathrm{bits} / \mathrm{s} / \mathrm{Hz}$ is obtained.

Figures 5 and 6 analyze the use of diversity combining. Figure 5 considers the effect of aperture diameter on the $\varepsilon$-outage spectral efficiency. It presents the spectral efficiency as a function of the normalized aperture $D / r_{0}$ for a constant phase coherence length $r_{0}$. In (a), MRC combining is employed. In (b), a SC combiner is considered. In all cases, the outage probability is small and fixed at $\varepsilon=0.001$, and the channel capacity per unit bandwidth is shown for different values of the number $L$ of combiner independent branches. The case $L=1$ corresponds to no receive diversity. The area $\pi D^{2}$ describes the combined, multi-aperture system equivalent aperture. When no receive diversity is considered, $D$ equals the receiver aperture diameter. If a $L$-aperture system is analyzed, each one of the aperture diameters equals $D / \sqrt{ } L$. For the smallest aperture considered, we assume $\gamma_{0}$ equal to 10 photons-per-symbol. For any other aperture diameter, the value of $\gamma_{0}$ is proportional to $D^{2}$. We study the outage channel capacity as a function of several parameters: the average signal-tonoise ratio (photons) per bit, the strength of atmospheric turbulence, the receiver aperture diameter $D$, and the number $\mathrm{L}$ of combiner branches. Again, turbulence effects are described by two parameters. Fried's coherence length $r_{0}$ describes the coherent diameter of the distorted wavefront phase. The scintillation index $\sigma_{\beta}$ describes the intensity of amplitude fluctuations. In our modeling, we have chosen typical values of these two parameters. Atmospheric fading leads to serious degradation in the receiver sensitivity measured in terms of received photons per symbol, resulting in either a higher error rate or a higher required transmit power for a given multilevel modulation technique. In general, the performance of an array signal-combining system should improve with an increasing number of receivers and, consequently, given a fixed collecting area, the combined system can offer superior performance. When a single $(L=1)$ or dual $(L=2)$ receiver system is considered, the existence of an optimal aperture diameter in coherent free-space links is apparent. This optimal aperture diameter maximizes the $\varepsilon$-outage capacity. When the aperture is larger than the optimal value, phase distortion cannot be overcome by the increase in collected power, and we observe a decrease in capacity. For $L>2$ receiver systems, or under strong scintillation conditions, no optimal value can be identified.

In Fig. 6, note the diminishing capacity returns that are obtained as the number of branches increases. Although as we increase $L$ the outage spectral efficiency improves appreciably, the greatest improvement is still obtained in going from single- to two-branch combining. In any case, even for such small outage probability $\varepsilon=0.001$, a multiple receiver system with $L=8$ apertures reach an outage spectral efficiency of over $10 \mathrm{bits} / \mathrm{s} / \mathrm{Hz}$, very close to the optimal spectral rate of a single branch AWGN channel as defined by Shannon. More detailed results and comments on our analysis will be presented at the meeting.

\section{CONCLUSIONS}

We have studied the impact of atmospheric turbulence-induced scintillation and phase aberrations on the performance of free-space optical links in which synchronous heterodyne receivers use modal wavefront compensation or diversity combining to mitigate the effects of atmospheric turbulence fading. We have defined a mathematical model for the signal received after propagation through the atmosphere and after modal compensation. By noting that the downconverted electrical signal current can be characterized as the sum of many contributions from different coherent regions within the aperture, we showed that the PDF of this signal can be described by a modified Rice distribution. The result is easily generalized to account for diversity combining techniques. The parameters describing the PDF depend on the turbulence conditions and the number of modes compensated at the receiver. We have separately quantified the effects of amplitude fluctuations and wavefront phase distortion on system performance, and have identified two different regimes of 
turbulence, depending on the receiver aperture diameter normalized to the coherence diameter of the wavefront phase. When the normalized aperture diameter is relatively small, amplitude scintillation dominates and, as phase fluctuations have little impact, performance is virtually independent of the number of modes compensated. When the normalized aperture is larger, amplitude fluctuations become negligible, and phase fluctuations become dominant, so that high-order phase compensation may be needed to improve performance to acceptable levels. We have found that for most typical link designs, wavefront phase fluctuations are the dominant impairment, and compensation of a modest number of modes can reduce performance penalties by several decibels.

Also, for typical turbulence conditions, sizeable gains in achievable rate are realizable by allowing for a rather small number of apertures to be combined. Furthermore, for a given probability of outage, there is a significant gain in the achievable rate when utilizing the MRC combiner over the SC combiner. This study allows for the optimization of channel capacity over a variety of turbulence conditions and for the selection of maximum code rates at a given probability of outage. These models are used to derive fundamental limits on outage probability and achievable rates for synchronous coherent communication links.

The research of Aniceto Belmonte was funded by the Spanish Department of Science and Technology MICINN Grant No. TEC2009-10025.

\section{REFERENCES}

1. D. L. Fried, "Optical heterodyne detection of an atmospherically distorted signal wave front," Proc. IEEE 55, 57-67 (1967).

2. D. L. Fried, “Atmospheric modulation noise in an optical heterodyne receiver," IEEE J. Quantum Electron. QE-3, 213-221 (1967).

3. R. J. Noll, "Zernike polynomials and atmospheric turbulence," J. Opt. Soc. Am. 66, 207-211 (1976).

4. A. Belmonte and J. M. Kahn, "Performance of synchronous optical receivers using atmospheric compensation techniques," Opt. Express 16, 14151-14162 (2008).

5. A. Belmonte and J. M. Kahn, "Capacity of coherent free-space optical links using diversity-combining techniques," Opt. Express 17, 12601-12611 (2009)

6. J. D. Parsons, "Diversity techniques in communications receivers," in Advanced Signal Processing, D. A. Creasey, ed. (Peregrinus, 1985), Chap. 6.

7. A. Belmonte and J. M. Kahn, "Capacity of coherent free-space optical links using atmospheric compensation techniques," Opt. Express 17, 2763-2773 (2009).

8. J. W. Strohbehn, T. Wang, and J. P. Speck, "On the probability distribution of line-of-sight fluctuations of optical signals," Radio Science 10, 59-70 (1975).

9. L.C. Andrews, R.L. Phillips, and C.Y. Hopen, Laser Beam Scintillation with Applications (SPIE Press, 2001) 


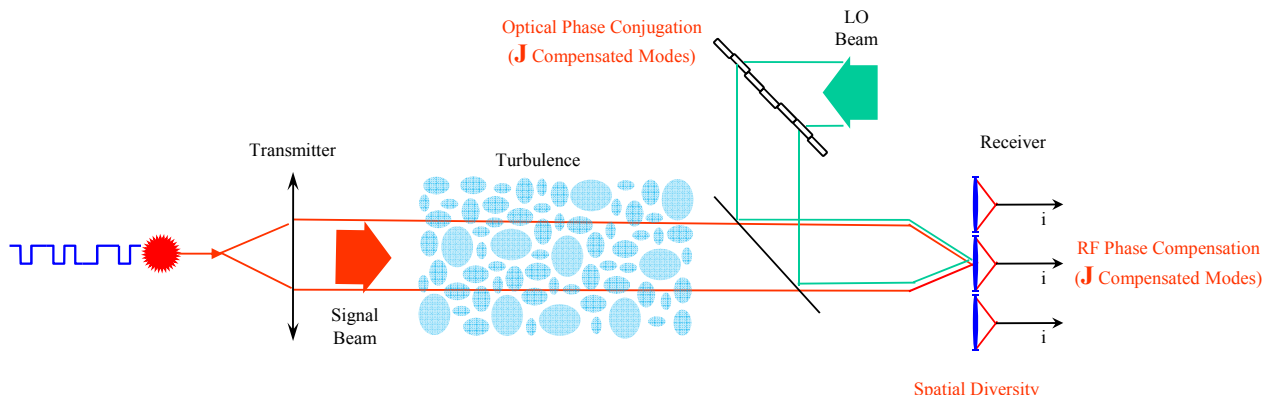

(L Apertures)

Fig. 1. A coherent free-space optical communication system is affected by atmospheric turbulence in two ways. First, turbulence induces a decrease of the mean received power level, which translates into a diminished signal-to-noise ratio in the receiver. Second, amplitude scintillation and phase distortion in the receiver plane act as intense sources of noise distorting the quality of the optical signal available for processing. These two effects combine to deteriorate the performance of the system. Phase compensation and diversity combining methods are two techniques that, at least in principle, can mitigate the effects of atmospheric turbulence on system performance.
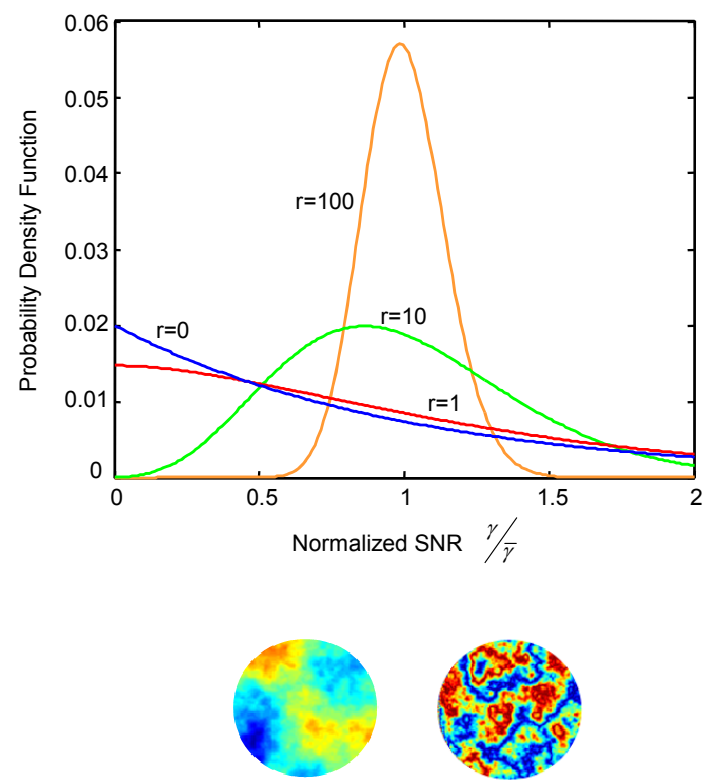

$\mathrm{r} \uparrow$

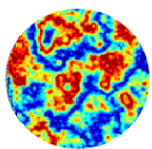

$\mathrm{r} \downarrow$

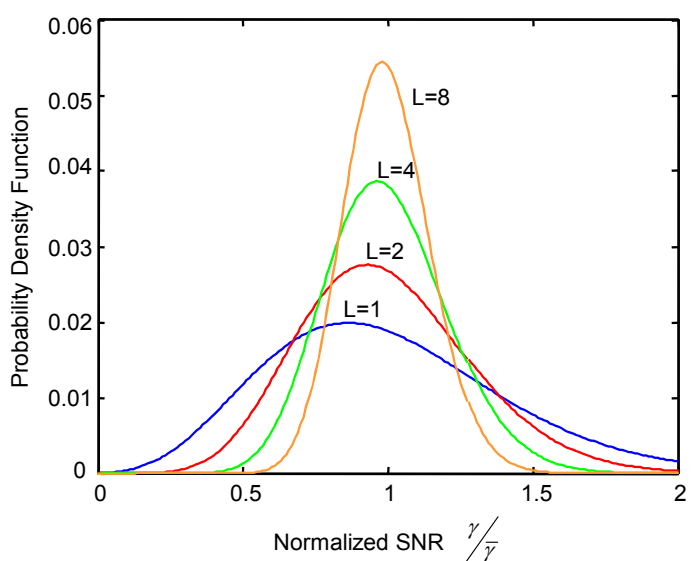

$\mathrm{t}_{0}$

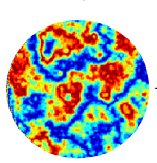

1

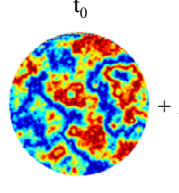

2 $\mathrm{t}_{0}$

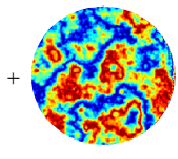

$\mathrm{L}$

Fig. 2. By noting that the downconverted signal current can be characterized as the sum of many contributions from different coherent regions within the aperture, we show that the probability density function (PDF) of this current can be well-approximated by a modified Rice distribution. In (a), we show the Rician density function as a function of the normalized SNR and the Rician factor $r$. In (b), we plot the PDF of the received SNR $\gamma_{\mathrm{MRC}}$ at the output of a perfect $L$-branch MRC coherent combiner in the atmosphere as a function of the normalized SNR and the number of combining branches $L$. 

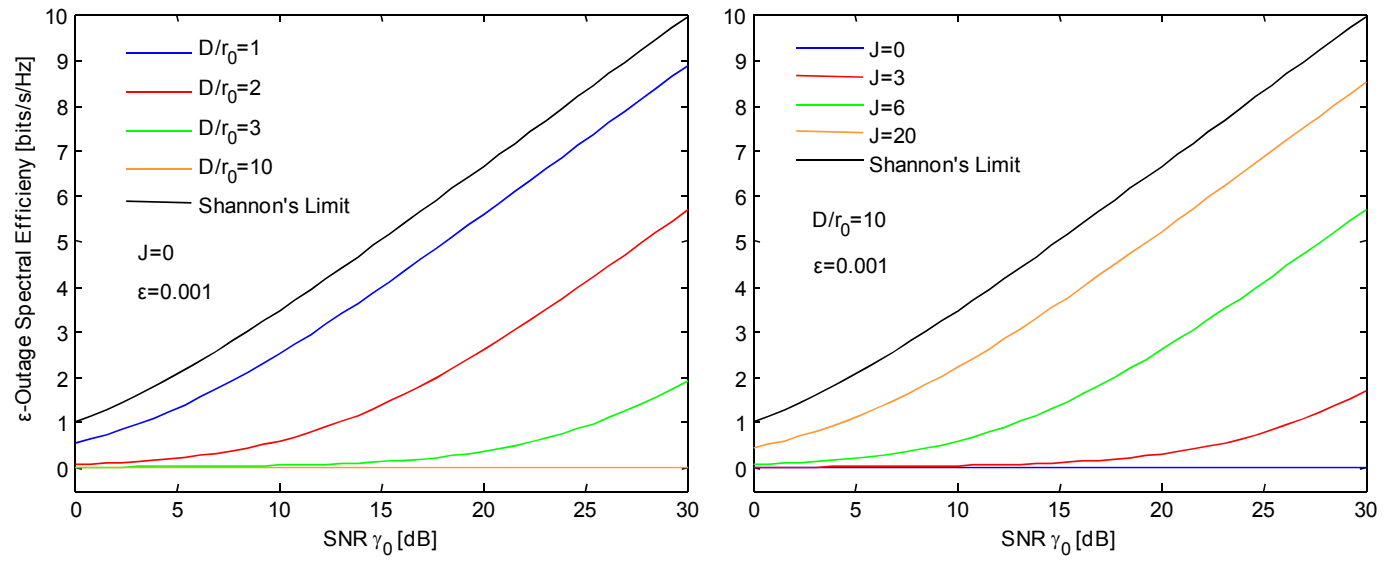

Fig. 3. $\varepsilon$-outage spectral efficiency vs. turbulence-free SNR per symbol $\gamma_{0}$ for coherent detection and additive white Gaussian noise (AWGN). Performance is shown for different values of: (a) the normalized receiver aperture diameter $D / r_{0}$, and (b) the number of modes $J$ removed by adaptive optics. The outage probability is fixed at $\varepsilon=0.001$. Amplitude fluctuations are neglected by assuming $\sigma_{\beta}{ }^{2}=0$. Turbulence is characterized by the phase coherence length $r_{0}$. In (a), $D / r_{0}$ ranges from 0.1 (weak turbulence) to 10 (strong turbulence). In (b), the compensating phases are expansions up to tilt $(J=3)$, astigmatism $(J=6)$, and 5 th-order aberrations $(J=20)$. The no-correction case $(J=0)$ is also considered. The AWGN Shannon limit is indicated by black lines.
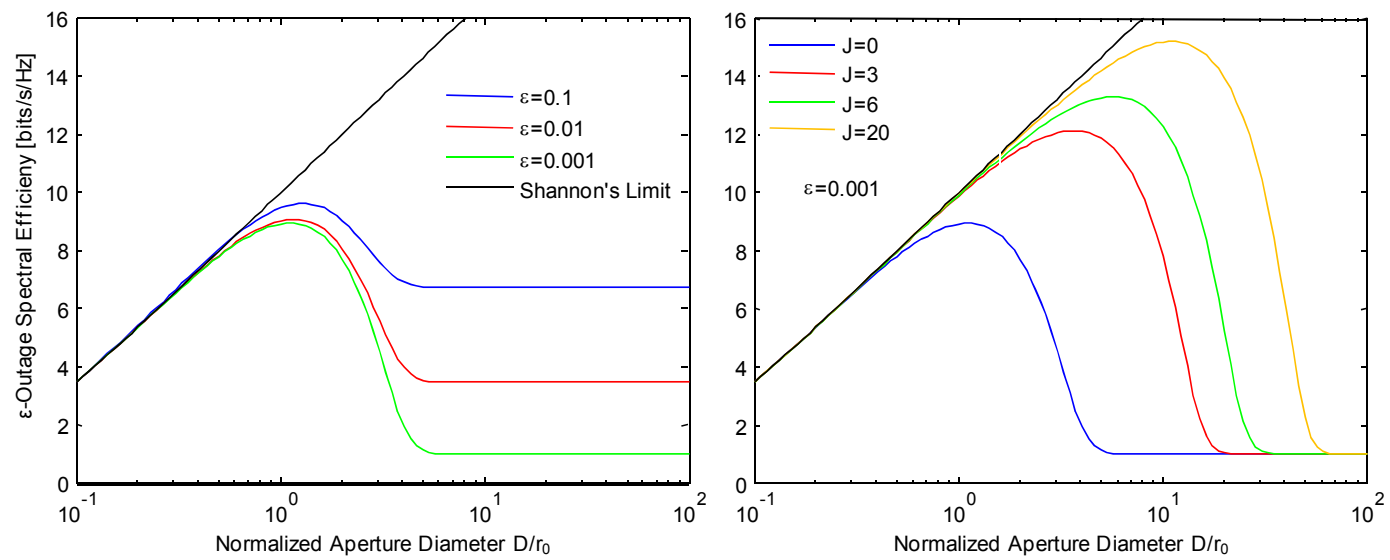

Fig. 4. $\varepsilon$-outage spectral efficiency vs. turbulence-free SNR per symbol $\gamma_{0}$ for coherent detection and additive white Gaussian noise (AWGN). Performance is shown for different values of: (a) the normalized receiver aperture diameter $D / r_{0}$, and (b) the number of modes $J$ removed by adaptive optics. The outage probability is fixed at $\varepsilon=0.001$. Amplitude fluctuations are neglected by assuming $\sigma_{\beta}{ }^{2}=0$. Turbulence is characterized by the phase coherence length $r_{0}$. In (a), $D / r_{0}$ ranges from 0.1 (weak turbulence) to 10 (strong turbulence). In (b), the compensating phases are expansions up to tilt $(J=3)$, astigmatism $(J=6)$, and 5 th-order aberrations $(J=20)$. The no-correction case $(J=0)$ is also considered. The AWGN Shannon limit is indicated by black lines. 

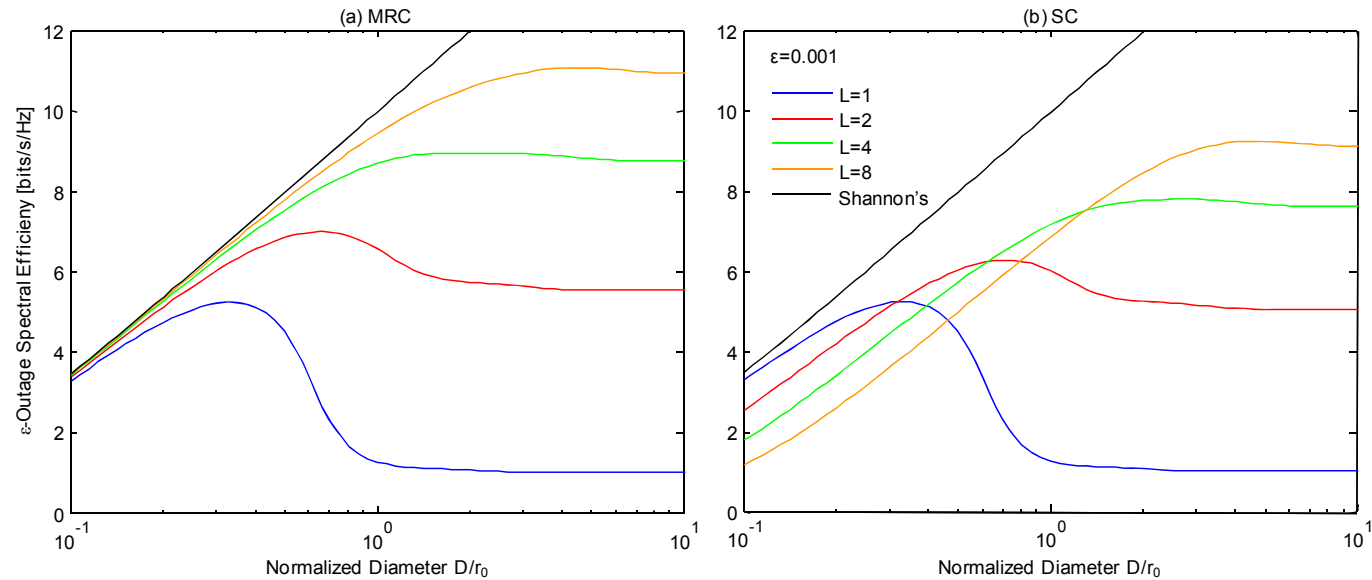

Fig. 5. $\varepsilon$-outage spectral efficiency vs. normalized receiver aperture diameter $D / r_{0}$ for coherent detection and AWGN. In (a), MRC combining is employed. In (b), a SC combiner is considered. In all cases, the outage probability is fixed at $\varepsilon=0.001$, and the channel capacity per unit bandwidth is shown for different values of the number $\mathrm{L}$ of combiner branches. The case $\mathrm{L}=1$ corresponds to no receive diversity (blue lines). The area $\pi \mathrm{D}^{2}$ describes the combined, multi-aperture system equivalent aperture. When no receive diversity is considered, $\mathrm{D}$ equals the receiver aperture diameter. The turbulence-free SNR per symbol $\gamma_{0}$ is proportional to the square of the aperture diameter $D$. For the smallest aperture considered, we assume $\gamma_{0}$ equal to 10 photons per symbol. In this plot, we neglect amplitude fluctuations by assuming $\sigma_{\beta}{ }^{2}=0$. In this case, turbulence is characterized by the phase coherence length $r_{0}$. The AWGN Shannon limit is indicated by black lines.
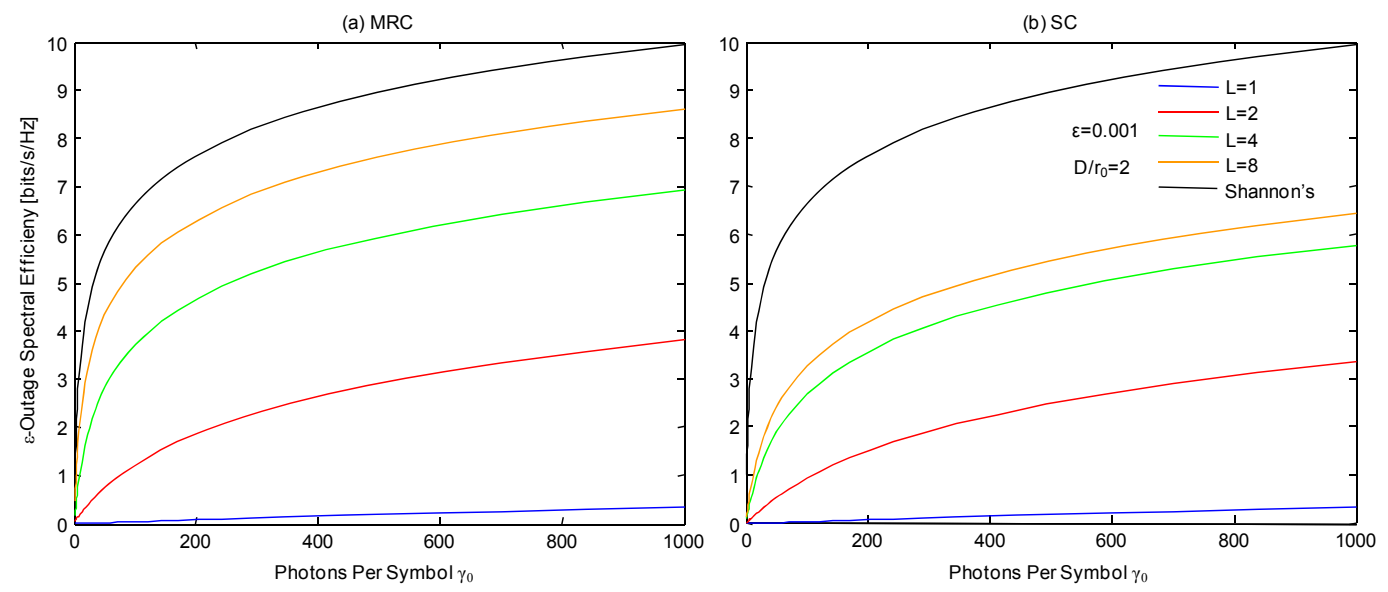

Fig. 6. $\varepsilon$-outage spectral efficiency vs. turbulence-free photons per symbol $\gamma_{0}$ for coherent detection and additive white Gaussian noise (AWGN). In (a), MRC combining is employed. In (b), a SC combiner is considered. In all cases, the outage probability is fixed at $\varepsilon=0.001$, and the channel capacity per unit bandwidth is shown for different values of the number $\mathrm{L}$ of combiner branches. The case $\mathrm{L}=1$ corresponds to no receive diversity (blue lines). Amplitude fluctuations are neglected by assuming $\sigma_{\beta}{ }^{2}=0$. Turbulence is characterized by a moderate phase coherence length $r_{0}$ such as $D / r_{0}=2$. The area $\pi \mathrm{D}^{2}$ describes the combined, multi-aperture system equivalent aperture. The AWGN Shannon limit is indicated by black lines. 